\title{
Molecular epidemiological study of feline coronavirus strains in Japan using RT-PCR targeting nsp14 gene
}

\author{
Yoshikazu Tanaka ${ }^{1{ }^{* \dagger}}$, Takashi Sasaki ${ }^{1 \dagger}$, Ryo Matsuda', Yosuke Uematsu ${ }^{2}$ and Tomohiro Yamaguchi ${ }^{2}$
}

\begin{abstract}
Background: Feline infectious peritonitis is a fatal disease of cats caused by infection with feline coronavirus (FCoV). For detecting or genotyping of FCoV, some RT-PCR plus nested PCR techniques have been reported previously. However, referring to the whole genome sequences (WGSs) registered at NCBI, there are no detection methods that can tolerate the genetic diversity among FCoV population. In addition, the quasispecies nature of FCoV, which consists of heterogeneous variants, has been also demonstrated; thus, a universal method for heteropopulations of FCoV variants in clinical specimens is desirable.

Results: To develop an RT-PCR method for detection and genotyping of FCoV, we performed comparative genome analysis using WGSs of 32 FCoV, 7 CCoV and 5 TGEV strains obtained from NCBI. As the PCR target, we focused on the nsp14 coding region, which is highly conserved and phylogenetically informative, and developed a PCR method targeting nsp14 partial sequences. Among 103 ascites, 45 pleural effusion and 214 blood specimens from clinically ill cats, we could detect FCoV in 55 (53.4\%), 14 (31.1\%) and 19 (8.9\%) specimens using the present method. Direct sequencing of PCR products and phylogenetic analysis allowed discrimination between type I- and II-FCoV serotypes. Our nsp14 amino acid sequence typing (nsp14 aa ST) showed that the FCoV clone with sequence type (ST) 42, which was the most predominant genotype of WGS strains, was prevalent in domestic cats in Japan.
\end{abstract}

Conclusions: Our nsp14 PCR scheme will contribute to virus detection, epidemiology and ecology of FCoV strains.

Keywords: Feline coronavirus, Epidemiology, Phylogenetic study

\section{Background}

Feline coronavirus (FCoV) can be classified into two biotypes, namely low virulent feline enteric coronavirus (FECV) and highly virulent feline infectious peritonitis virus (FIPV) [1]. Clinical appearance of FECV, if any, is characterized by mild enteritis. In contrast, FIPV efficiently replicates in macrophages/monocytes, and can lead to FIP, which is a highly lethal systemic granulomatous disease [2]. FIPV exists in two serotypes based on virus neutralizing antibodies, type I and type II $[1,3]$. Serotype I virus has a distinctive spike protein, while the spike protein of serotype II is a recombinant protein between feline and canine enteric coronaviruses [4]. Type I

\footnotetext{
*Correspondence: ytanaka@nvlu.ac.jp

'Equal contributors

'Department of Veterinary Hygiene, Veterinary School, Nippon Veterinary \& Life Science University, 1-7-1 Kyounan, Musashino, Tokyo 180-8602, Japan Full list of author information is available at the end of the article
}

FECVs/FIPVs predominate throughout the world, but type II strains appear to be more adaptable to tissue culture. However, type I strains are more likely to cause clinical FIP signs [3]. FCoV is also common in healthy cats worldwide; less than $10 \%$ of FCoV seropositive cats develop FIP [5-9]. Therefore, measuring antibody levels against FCoV is rarely of diagnostic value in FIP. Thus, Histopathological examination of infected tissues is needed for the aetiological diagnosis of FIP $[10,11]$.

RT-PCR plus nested PCR and real-time PCR techniques have allowed the detection or genotyping of FCoV $[3,12-15]$. However, they have not been fully reevaluated whether they can tolerate the genetic diversity of FCoV. In recent years, whole genome sequences of clinical strains of $\mathrm{FCoV}$ have been registered in the NCBI database by Rottier et al. at the J. Craig Venter Institute, and we have been able to readily obtain nucleotide sequences of the FCoV genome. With regard 
to genome information, there have been no reports on PCR-based methods that can tolerate the genetic diversity in whole genome sequenced FCoV strains. Thus, a detection method for all variants of $\mathrm{FCoV}$ is highly desirable.

In the present study, in order to construct a universal method for FCoV variants, we performed a genomewide analysis of $\mathrm{FCoV}$, and developed an RT-PCR method for detecting $\mathrm{FCoV}$ in clinical specimens. Consequently, direct sequencing of PCR products and phylogenetic analysis allowed discrimination between type I and II serotypes of FCoV. Using this method, we investigated the population genetics of $\mathrm{FCoV}$ strains from diseased cats in Japan.

\section{Methods}

Bioinformatics for FCoV, CCoV and TGEV

As shown in Additional file 1, thirty-two FCoV strains, seven canine coronavirus (CCoV) strains, four transmissible gastroenteritis coronavirus (TGEV) strains, a porcine respiratory coronavirus (PRCV) strain and a Mink coronavirus $(\mathrm{MiCoV})$ strain were used for comparative genome analysis. All sequences were obtained from the NCBI database. Gene searches and annotation were carried out by GeneMarkS (http://opal.biology.gatech.edu/ genemarks.cgi) [16], RAST annotation server (http://rast. nmpdr.org) [17] and the blast-based method. MAFFT v7.037 was used for multiple sequence alignment [18], and Aminosan v1.0.2011 was performed for amino-acid substitution model selection [19]. Phylogenetic inference using maximum likelihood (ML) and bootstrapping was performed using MEGA v5.05 [20].

In order to perform a genome-wide comparison among type I- and II-FCoV, CCoV and TGEV strains, we used the Genotyping tool at NCBI (http://www.ncbi.nlm. nih.gov/projects/genotyping/formpage.cgi), which helps identify genotype and recombinant sequences using the blast-based method [21]. Default values were used; 300 for "window" and 100 for "increment".

\section{Cell culture and virus}

Felis catus whole fetus-4 (fcwf-4; American Type Culture Collection, VA, USA) cells were maintained in Dulbecco's modified Eagle's medium (D-MEM, SigmaAldrich, Tokyo, Japan) supplemented with $10 \%$ fetal bovine serum (JRH, Nissui, Tokyo, Japan). We purified FCoV using linear sucrose gradient ultracentrifugation from FCoV 79-1146 strains (a gift from Tsutomu Hodatsu, Kitasato University, Japan) propagated in fcwf-4 cells.

\section{RNA extraction and reverse transcription-PCR}

Isogen-LS (Nippon Gene, Toyama, Japan) was used for RNA preparation from clinical specimens (whole blood, pleural fluid, ascites, pericardial effusion and cerebral fluid), and fcwf-4 cells infected with FCoV (79-1146 strain) according to the manufacturer's protocol. Total RNA was reverse-transcribed using the PrimeScript RTPCR kit (Perfect Real Time; Takara Bio, Shiga, Japan), as reported previously [22].

\section{Construction of PCR method for detection and genotyping of FCoV strains}

In order to construct a PCR method that detects variants in FCoV strains, primers were designed by multiple alignments of nucleotide sequences of the nsp14 genes in all whole genome-sequenced $\mathrm{FCoV}$, closely related subspecies, CCoV and TGEV strains. The primer set nsp14-F (5'-GTGATGCTATCATGACTAG$\left.3^{\prime}\right)$ and nsp14-R (5'-CACCATTACAACCTTCTAA3') was used. The expected size of PCR products was $417 \mathrm{bp}$. The reaction mixture for PCR consisted of 4 $\mu \mathrm{l}$ of cDNA in a total volume of $25 \mu \mathrm{l}$ composed of 1 $\mathrm{U}$ of Ex Taq (Takara-Bio), $10 \mathrm{pmol}$ of each primer, 0.2 $\mathrm{mM}$ deoxynucleoside triphosphate mixture and $1 \times$ reaction buffer (Takara-Bio). Reaction mixtures were thermally cycled once at $95^{\circ} \mathrm{C}$ for $2 \mathrm{~min} ; 40$ times at $95^{\circ} \mathrm{C}$ for $30 \mathrm{~s}, 48^{\circ} \mathrm{C}$ for $35 \mathrm{~s}$, and $72^{\circ} \mathrm{C}$ for $45 \mathrm{~s}$; and then once at $72^{\circ} \mathrm{C}$ for $5 \mathrm{~min}$. Using $6 \mu \mathrm{l}$ of PCR sample, DNA fragments were analyzed by electrophoresis in $1 \times$ Tris-acetate-EDTA on a $1 \%$ agarose gel stained with ethidium bromide. In addition, these PCR products were directly sequenced using a Big Dye terminator (version 3.1) cycle sequencing kit (Applied Biosystems, Tokyo, Japan) with an ABI Prism 3100 genetic analyzer (Applied Biosystems).

Total RNA was extracted from the fcwf-4 cells infected with FCoV strain 79-1146, and was reversetranscribed into cDNA. Viral cDNA was quantified using a real-time PCR method, as reported previously [22]. Using cDNA samples of known copy numbers, we evaluated the detection limit of our PCR method targeting nsp14.

\section{Study population in molecular epidemiological study of FCoV strains from clinically ill cats in Japan}

In the period between 2007 and 2014 in Japan, 372 specimens (103 ascites, 45 pleural effusion, 214 blood, 9 cerebral fluid and 1 pericardial effusion), which were obtained in the examination of clinically ill cats for the presence of $\mathrm{FCoV}$, were used in the present study. To detect $\mathrm{FCoV}$ in clinical specimens, we performed RTPCR using a random primer plus a single PCR targeted nsp14 constructed in the present study. To differentiate type I-FCoV from type II-FCoV or CCoV genotypes, direct sequencing of their PCR products and phylogenetic analysis were carried out. 
In order to determine the significance of differences in detection rate between ascites, pleural effusion and blood specimens, relative risk and odds ratio were calculated using MedCalc software (http://www.medcalc.org/ index.php).

nsp14 amino acid sequence typing (nsp14 aa ST) and comparison of genetic diversity in $\mathrm{FCoV}$ strains between different specimens

In order to devise a genotyping method for FCoV, we assigned an ST number to all distinct nsp14 partial amino acid sequences (135 aa) found in all FCoV strains, which were sequenced in the present study and previously registered in the NCBI database. We compared the population genetic structures of FCoV strains in Japan with whole genome-sequenced strains from 6 different countries.

\section{Comparison of diversity and evenness indexes of FCoV strains by specimen}

The diversity and evenness of ST distribution by specimen type were calculated using Simpson's diversity index $(1-\lambda)$ and Pielou's evenness index $\left(J^{\prime}\right)[23,24]$. These parameters have generally been used for comparisons of biodiversity between geographically or ecologically separated environments. Both values range from 0 (no diversity or evenness) to 1 (extreme diversity or evenness).

\section{Results}

Genome-wide comparison between FCoV strains and closely related subspecies, CCoV and TGEV

In order to compare the genome structures between type I-FCoV and type II-FCoV, and the closely related subspecies CCoV, TGEV and PRCV, we carried out a blast-based genome-wide comparison. After performing genotyping, genomic regions of $300 \mathrm{bp}$ in each viral strain were color coded according to scores (0 to 300) based on nucleotide similarities against $\mathrm{FCoV}$ strain UU9 (Figure 1). In the genome structures of all strains with type II-FCoV genotype, events of large-scale recombination were found in a locus that stretches for about $12,000 \mathrm{bp}$ from the first half of nsp12 to upstream of the nucleocapsid gene, as reported previously $[25,26]$. The recombination site varied slightly from one strain to another. In the phylogenetic tree based on concatenated sequences of nsp13, nsp14, nsp15, nsp16 and spike, which are protein-coding regions located within recombinant sequences, all type II-FCoV strains were clustered into a clade belonging to canine coronavirus strains (data not shown). Among the alpha-coronavirus 1 subspecies, to which FCoV, CCoV, TGEV and PRCV belonged, the coding regions from nsp14 to nsp16 were the most highly conserved (Figure 1).

\section{PCR method targeting nsp14 for detection of FCoV strains from clinical specimens}

Based on a genome-wide comparison using the Genotyping tool at NCBI, we focused on protein-coding sequences within nsp14 to nsp16 as a candidate PCR target. The sequence region was suited to universal detection of variants because of its highly conserved sequence. Among the primer sets designed in nucleotide sequences conserved in all $\mathrm{FCoV}, \mathrm{CCoV}$, TGEV and PRCV strains, we determined the best primer set, nsp14-F and nsp14-R, which allowed amplification of $417 \mathrm{bp}$ of the nsp14 partial sequences. Consequently, this PCR is universally applicable to all alpha-coronavirus 1 subspecies.

We were able to detect FCoV strains from ascites, pleural effusion, pericardial effusion and blood samples using the present method. Few nonspecific bands were found in agarose gel electrophoresis (Figure 2). Specific amplification of target sequences against all PCR products was confirmed by sequencing analysis. To evaluate the detection limit, the present method was applied to cDNA samples of known copy number using PCR. The detection limit was $4.59 \times 10^{2}$ copies $/ \mathrm{mL}$.

\section{Sequence-based differentiation of type I-FCoV from type II-FCoV or CCoV}

The nsp14 coding region was located on the recombination hotspot in type II-FCoV, and sequencing analysis of the region permitted discrimination between type I and II serotypes. The nucleotide identity of the nsp14 partial sequence among FCoV, CCoV, TGEV and PRCV strains ranged from 89.9 to $100 \%$. By phylogenetic analysis based on nsp14 partial nucleotide sequences (406 bp), we were able to successfully distinguish the type IFCoV genotype from type II-FCoV, CCoV or TGEV (Figure 3). Unfortunately, our method had no discriminating power for differentiating between type II-FCoV and $\mathrm{CCoV}$ genotypes.

\section{Comparison of FCoV detection rate from ascites, pleural effusion and blood in cats}

Using the PCR and direct-sequencing method targeting nsp14 in the present study, we surveyed FCoV strains from clinically ill cats on which FIP was suspected but not confirmed by a complete clinico-pathological or pathological workup in veterinary hospitals in Japan. In peritoneal and pleural fluid samples, FCoV was detected in 55 of 103 (53.4\%) and 14 of 45 (31.1\%), respectively. All of these exhibited the type I-FCoV genotype. In a comparison of positivity rate of $\mathrm{FCoV}$ between peritoneal and pleural effusion samples, relative risk and odds ratio were 


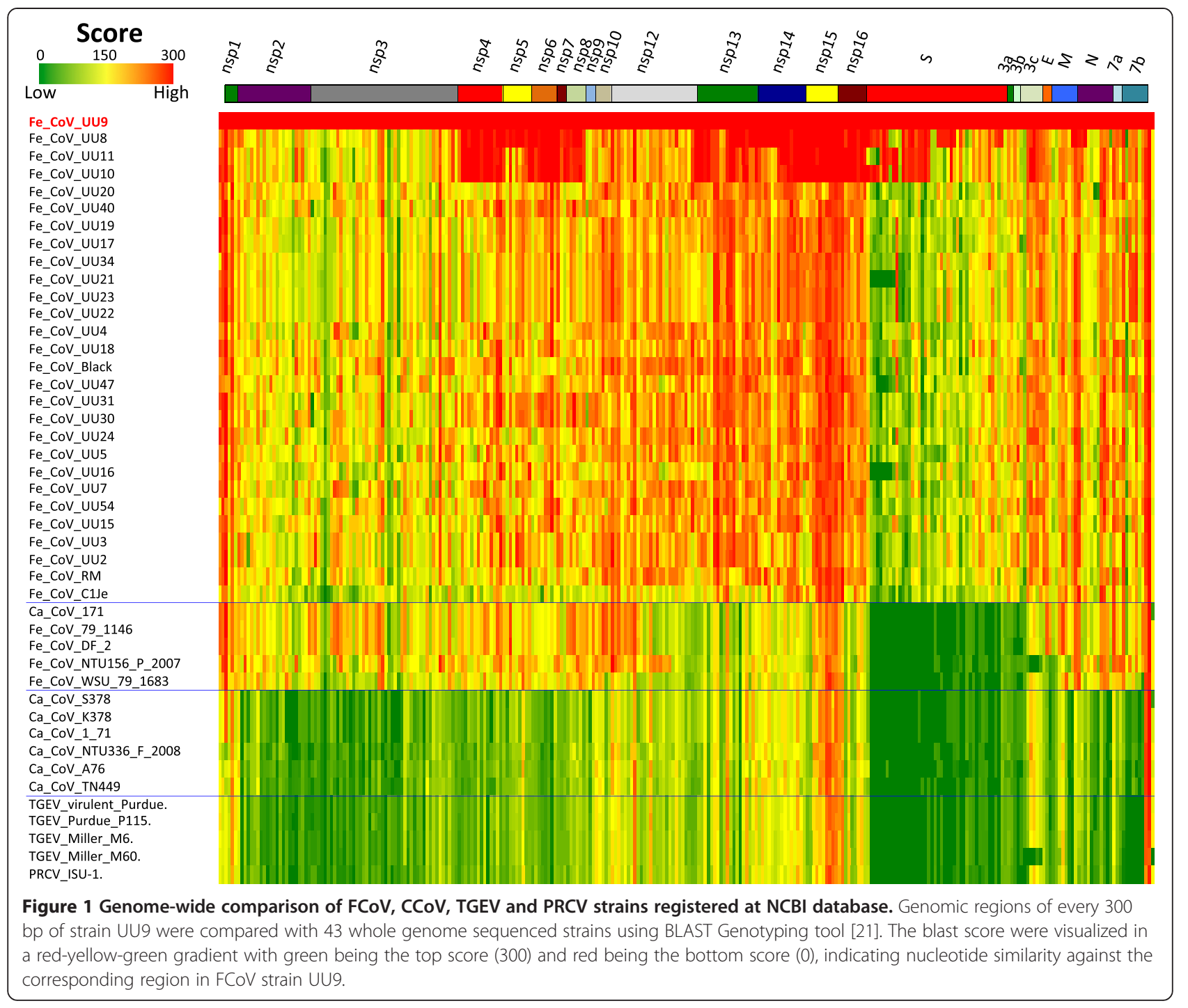

1.7164 and $2.5372(95 \%$ confidence interval $=1.21-5.32, \mathrm{z}$ statistic $=2.465, \mathrm{P}=0.0137)$, respectively, and we found statistically significant differences between the two.

On the other hand, positive results on nsp14 PCR from 214 blood samples, 9 cerebral fluid samples and 1 pericardial effusion sample from 224 clinically ill cats, were seen in $19(8.9 \%), 0(0 \%)$ and $1(100 \%)$, respectively. Only one of the blood samples exhibited type II-FCoV or $\mathrm{CCoV}$ genotype.

\section{Population genetics of FCoV strains in Japan by nsp 14 amino acid sequence typing (nsp14 aa ST)}

Using nsp 14 amino acid sequence typing (nsp14 aa ST), which was an allelic analysis based on 135 amino acid residue of nsp14, we identified fifty-three unique STs among the $89 \mathrm{FCoV}$-positive samples in this study, and 39 whole genome-sequenced $\mathrm{FCoV}$ and $\mathrm{CCoV}$ strains registered in the NCBI database (Additional file 2) [27-29]. The assigned ST numbers of nsp14 aa ST in whole genomesequenced strains are shown in Additional file 1.

As shown in Figure 4, our nsp14 aa ST indicated that the most predominant genotype among domestic cats in Japan was ST42 (24 of 89, 27.0\%), which was also found most frequently in whole genome-sequenced strains, followed by ST8 $(n=10)$, and ST25 $(n=7)$, and the top three STs accounted for $46.1 \%$ of total FCoV-positive samples. Among ascites specimens in the present study, ST42 was the most frequent genotype $(n=18)$, followed by ST8 $(n=5)$ and ST25 $(n=5)$. Of pleural effusion and blood samples, multiple strains of ST42 $(n=4)$, ST8 $(\mathrm{n}=2)$ and ST8 $(\mathrm{n}=3)$, ST25 $(\mathrm{n}=2)$, ST42 $(\mathrm{n}=2)$ were identified, respectively. We were unable to find any correlations between types of specimen and STs.

As shown in Table 1, populations of FCoV strains from blood specimens showed high values for both diversity and evenness indexes, which indicates an almost random 


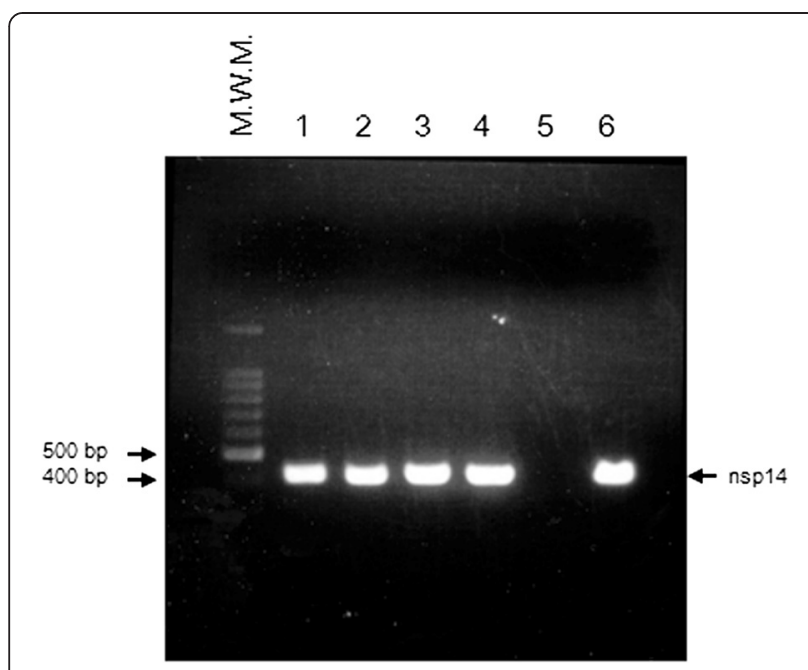

Figure 2 Agarose gel electrophoresis of nsp14 PCR amplification products from clinical specimens. Lane 1; pleural fluid, lane 2; ascites, lane 3; pericardial effusion, lane 4; FCoV positive whole blood by a qPCR method, lane 5; FCoV negative whole blood by a qPCR method, lane 6; fcwf-4 cells infected with FCoV strain 79-1146. M.W.M. (molecular weight marker; $100 \mathrm{bp}$ ladder) is loaded on the agarose gel.

pattern. The lowest values for both diversity and evenness indexes in ascites suggested the presence of ascites-tropic clones. In addition to $\mathrm{FCoV}$ detection rate, population structures of FCoV strains between ascites and pleural effusion specimens also exhibited different trends.

\section{Discussion}

In previous epidemiological studies, nested PCR techniques targeting 3 '-UTR and spike sequences have been widely used for viral detection and discrimination between type I and II serotypes, respectively [3,12,30-36]. However, previous methods are time-consuming, and nonspecific amplification often occurs in the course of nested PCR. Our nsp14 PCR method allowed detection of FCoV using a single PCR, and little nonspecific amplification occurred during PCR in various clinical specimens, suggesting that the primer set exhibited high specificity to only viral genome. Consequently, our method allows to ascertain accurately the presence of FCoV in any type of specimen without confirmation by sequencing of PCR products. Recently, Soma et al. reported that positivity of FCoV was $44.1 \%$ (377 of 854 ) in ascites from cats suspected of wet FIP in Japan using the 3'-UTR nested PCR method [36]. Although a simple comparison was not clear because of different study populations, detection sensitivity of FCoV in ascites specimens of our nsp14 single PCR was comparable or better $(P=0.0746)$, compared with the study using the nested PCR method. There have been some reports on the quasispecies in FCoV [31,37-41]; Battilani et al. reported that quasispecies composition is correlated with the seriousness of clinical form and lesions in the organs [37]. Therefore, detection of a population that consists of a complex of heterogeneous variants is necessary in the diagnosis of FCoV infection. Consequently, this may have resulted in improved sensitivity for FCoV detection by the present PCR targeting nsp14, which is a highly conserved region among alpha-coronavirus1 subspecies. The present method is the most reliable PCR method that can tolerate the genetic diversity of FCoV.

Using the scheme of nsp14 aa ST, we found that a clone of FCoV, ST42, is prevalent in domestic cats in Japan. It is likely that the ST42 FCoV clone is also endemic worldwide, as it accounts for most of the whole genome-sequenced strains registered at NCBI. The global distribution of ST8 and ST25 FCoV clones, which are second and third most common STs after ST42 in Japan, remains to be clarified. Further epidemiological study is thus needed.

Aggregated distribution of specific STs in FCoV strains from ascites was in contrast to that of blood specimens that formed an almost random pattern, suggesting that a tropic ascites clone is present among FCoV populations. A previous study also reported differences of quasispecies compositions of FCoV in ORF7b and $\mathrm{N}$ region between organs [38]. Interestingly, diversity and evenness of FCoV strains from pleural effusion were similar to those of blood but not ascites. With regard to both viral detection rate and population structures, different trends between ascites and pleural effusion specimens suggest that pathology of FCoV relevant-pleural effusion or pleural FIP have markedly different characteristics from those of FCoV relevant-ascites or peritoneal FIP. Previous studies reported that genomic alteration or variability of viral population during infection could affect the organ-specificity, severity and immunological escape $[37,39,41]$. Although, an onset of FCoV infection or FIP highly depends on host factors, FCoV also might exhibit the pathogenic diversity by viral strains.

The so-called internal mutation hypothesis, which postulates that viruses transition from avirulent to virulent via certain mutations leading to FIP pathogenesis, is generally believed in veterinary medicine [10]. However, there have been no reports on the identification of consensus mutations in any FCoV strains; no relationship between viral phylogeny and virulence has previously been found [42-44]. Our diversity analysis did not search for mutations in the FCoV genome responsible for FIP pathogenesis, suggesting that genetically diverse FCoV clones are present in domestic cats and that dynamically selected clones can cause FCoV-related ascites or wet FIP. Other reports also suggested that sequential emergence of variants and replacing the pre-existing population occurred in FCoV under the host immune 


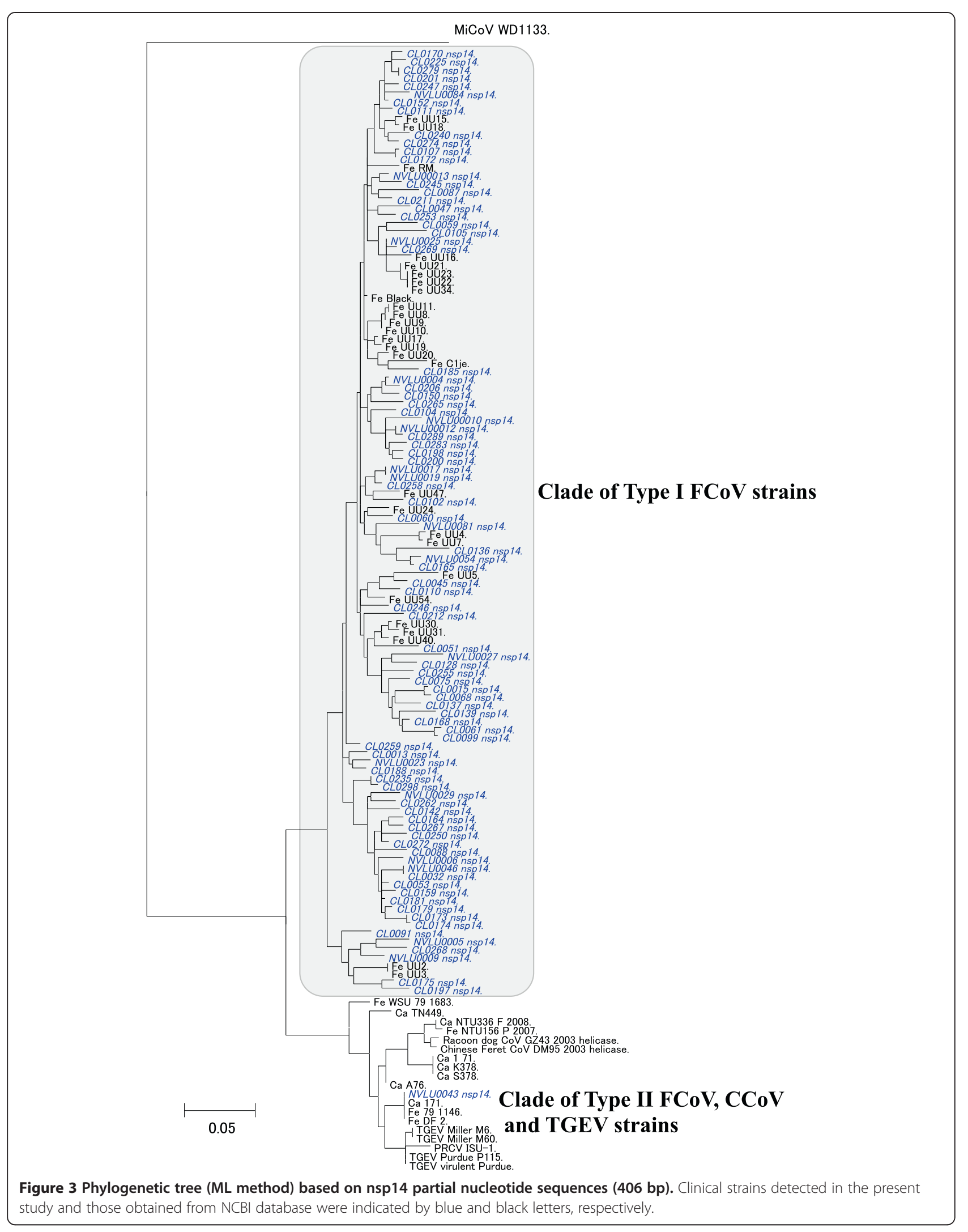




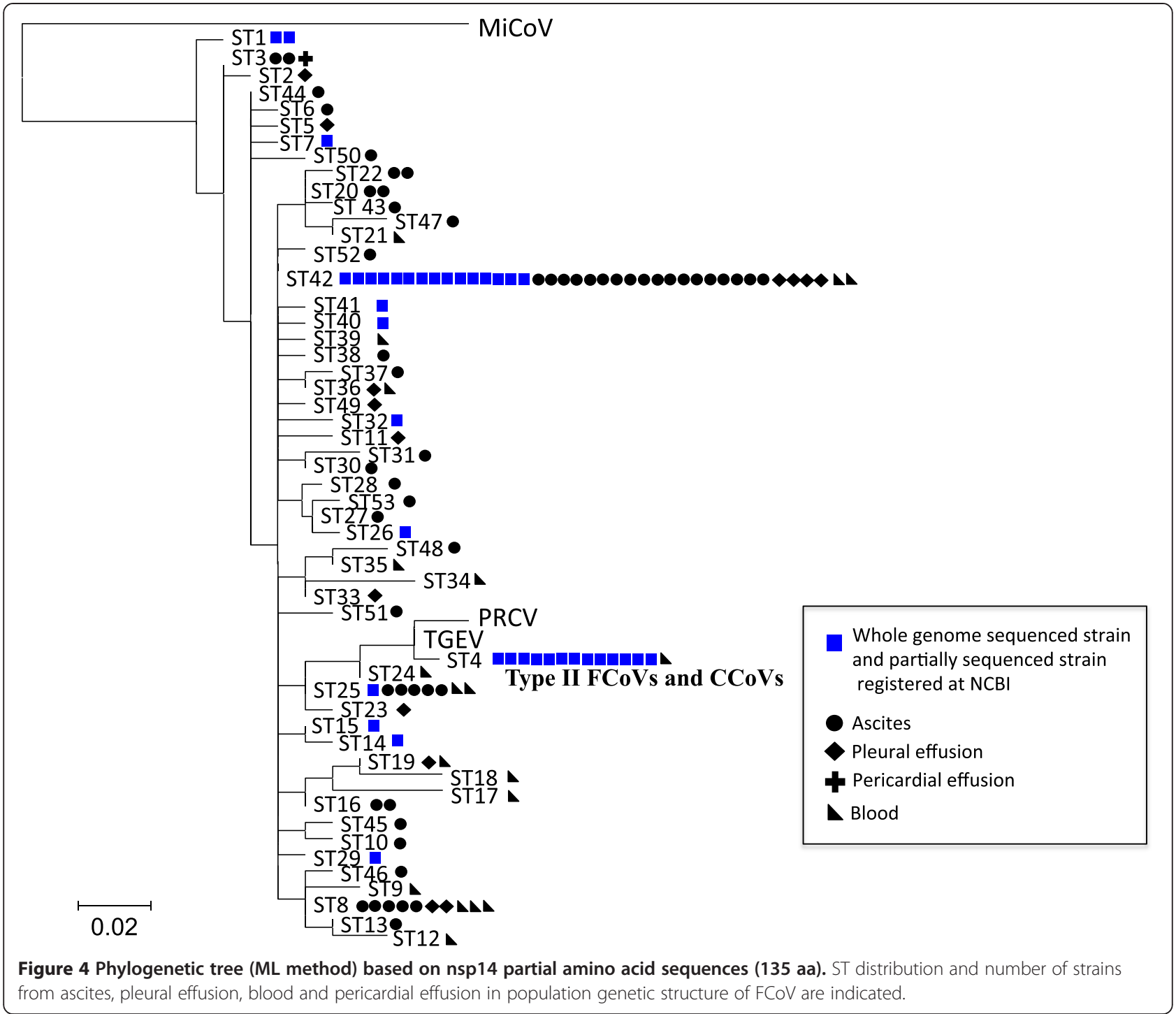

pressure $[39,41]$. Thus, transition to FIP may occur through changes in viral populations in a feline host rather than internal mutations.

Recently, severe acute respiratory syndrome (SARS) in 2002/2003 and Middle East respiratory syndrome (MERS) in 2012 have emerged as human infectious disease from zoonotic coronaviruses $[45,46]$. To trace the original infectious source of coronavirus zoonotic transmission, we need to understand the ecology and population structures of viral strains in various animal species. Although there have been reports on detection of strains with the FCoVgenotype in host species of Carnivora other than domestic cats [35,47-49], our ecological understanding of this virus remains insufficient. The present method, which was applicable to all alpha-coronavirus1 subspecies, will also contribute to our ecological understanding of this virus.

\section{Conclusions}

In conclusion, we developed a detection and genotyping tool for all variants of $\mathrm{FCoV}$, and confirmed the presence

Table 1 Diversity and evenness indexes of FCoV strains by specimens

\begin{tabular}{lllllll}
\hline Kind of specimen & No. of specimens & No. of strains & No. of STs & Simpson's index (1- $\boldsymbol{\lambda})$ & Pielou's index & Predominant ST(s) \\
\hline Ascites & 103 & 55 & 26 & 0.881 & 0.661 \\
Pleural effusion & 45 & 14 & 10 & 0.923 & 0.812 \\
Blood & 214 & 19 & 15 & 0.971 & 0.895 \\
\hline
\end{tabular}

${ }^{\mathrm{a}} \mathrm{ST}(\mathrm{s})$ which accounted for not less than $10 \%$ of clones in the population. 
of an endemic FCoV clone, ST42, in Japan and probably worldwide. The present nsp14 PCR method will contribute to molecular epidemiology and ecological findings in alpha-coronavirus 1 subspecies, including FCoV.

\section{Additional files}

Additional file 1: Coronavirus strains for whole genome analysis used in the present study. Thirty-two FCoV strains, seven canine coronavirus (CCoV) strains, four transmissible gastroenteritis coronavirus (TGEV) strains, a porcine respiratory coronavirus (PRCV) strain and a Mink coronavirus (MiCoV) strain were used for comparative genome analysis. The assigned ST numbers of nsp14 aa ST in whole genome-sequenced strains are shown.

Additional file 2: Amino acid sequences of each ST in nsp14 aa ST. Amino acid sequences, representative strains and the accession numbers of fifty-three unique STs in nsp14 aa ST are shown in this table. The sequences of representative strains detected in the present study have been deposited in the DDBJ/GenBank database.

\section{Abbreviations}

FCoV: Feline coronavirus; WGSs: Whole genome sequences; nsp14 aa ST: nsp14 amino acid sequence typing; ST: Sequence type; FECV: Feline enteric coronavirus; FIPV: Feline infectious peritonitis virus; CCoV: Canine coronavirus; TGEV: Transmissible gastroenteritis coronavirus; PRCV: Porcine respiratory coronavirus; MiCoV: Mink coronavirus; ML: Maximum likelihood; SARS: Severe acute respiratory syndrome; MERS: Middle East respiratory syndrome; M.W.M: Molecular weight marker.

\section{Competing interests}

The authors declare that they have no competing interests.

\section{Authors' contributions}

YT and ST carried out biochemical experiments, participated in data collection and analysis, and prepared the manuscript. RM carried out PCR and determination of the sequence of nsp14. YU and TY provided most specimens and performed real-time qPCR detections. All authors read and approved the final manuscript.

\section{Acknowledgements}

This study was supported by a Grant-in-Aid for Scientific Research (C) from the Japan Society for the Promotion of Science and the Strategic Research Base Development Program for Private Universities from the Ministry of Education, Culture, Sports Science and Technology of Japan (MEXT), 2008-2012.

\section{Author details}

'Department of Veterinary Hygiene, Veterinary School, Nippon Veterinary \& Life Science University, 1-7-1 Kyounan, Musashino, Tokyo 180-8602, Japan. ${ }^{2}$ Canine Lab., Inc., Nokodai-Tamakoganei Venture Port 302, 2-24-16, Koganei, Tokyo 184-0012, Japan.

Received: 21 June 2014 Accepted: 24 February 2015

Published online: 11 March 2015

\section{References}

1. Addie D, Belak S, Boucraut-Baralon C, Egberink H, Frymus T, Gruffydd-Jones $T$, et al. Feline infectious peritonitis. ABCD guidelines on prevention and management. J Feline Med Surg. 2009;11:594-604.

2. Fiscus SA, Teramoto YA. Antigenic comparison of feline coronavirus isolates: evidence for markedly different peplomer glycoproteins. J Virol. 1987;61:2607-13.

3. Kummrow M, Meli ML, Haessig M, Goenczi E, Poland A, Pedersen NC, et al. Feline coronavirus serotypes 1 and 2: seroprevalence and association with disease in Switzerland. Clin Diagn Lab Immunol. 2005:12:1209-15.

4. Shiba N, Maeda K, Kato H, Mochizuki M, Iwata H. Differentiation of feline coronavirus type I and II infections by virus neutralization test. Vet Microbiol. 2007;124:348-52
5. Addie DD. Clustering of feline coronaviruses in multicat households. Vet J. 2000;159:8-9.

6. Addie DD, Jarrett O. A study of naturally occurring feline coronavirus infections in kittens. Vet Rec. 1992:130:133-7.

7. Brown MA, Troyer JL, Pecon-Slattery J, Roelke ME, O'Brien SJ. Genetics and pathogenesis of feline infectious peritonitis virus. Emerg Infect Dis. 2009;15:1445-52

8. Kennedy M, Citino S, McNabb AH, Moffatt AS, Gertz K, Kania S. Detection of feline coronavirus in captive Felidae in the USA. J Vet Diagn Invest. 2002;14:520-2

9. Pedersen NC. A review of feline infectious peritonitis virus infection: 1963-2008. J Feline Med Surg. 2009;11:225-58.

10. O'Brien SJ, Troyer JL, Brown MA, Johnson WE, Antunes A, Roelke ME, et al. Emerging viruses in the Felidae: shifting paradigms. Viruses. 2012:4:236-57.

11. Sharif S, Arshad SS, Hair-Bejo M, Omar AR, Zeenathul NA, Alazawy A. Diagnostic methods for feline coronavirus: a review. Vet Med Int. 2010:7.

12. Addie DD, Schaap IA, Nicolson L, Jarrett O. Persistence and transmission of natural type I feline coronavirus infection. J Gen Virol. 2003;84:2735-44.

13. Gut M, Leutenegger CM, Huder JB, Pedersen NC, Lutz H. One-tube fluorogenic reverse transcription-polymerase chain reaction for the quantitation of feline coronaviruses. J Virol Methods. 1999;77:37-46.

14. Herrewegh AA, de Groot RJ, Cepica A, Egberink HF, Horzinek MC, Rottier PJ. Detection of feline coronavirus RNA in feces, tissues, and body fluids of naturally infected cats by reverse transcriptase PCR. J Clin Microbiol. 1995:33:684-9

15. Hornyak A, Balint A, Farsang A, Balka G, Hakhverdyan M, Rasmussen TB, et al. Detection of subgenomic mRNA of feline coronavirus by real-time polymerase chain reaction based on primer-probe energy transfer (P-sg-QPCR). J Virol Methods. 2012;181:155-63.

16. Besemer J, Lomsadze A, Borodovsky M. GeneMarkS: a self-training method for prediction of gene starts in microbial genomes. Implications for finding sequence motifs in regulatory regions. Nucleic Acids Res. 2001;29:2607-18.

17. Aziz RK, Bartels D, Best AA, DeJongh M, Disz T, Edwards RA, et al. The RAST Server: rapid annotations using subsystems technology. BMC Genomics. 2008;9:75.

18. Katoh K, Standley DM. MAFFT multiple sequence alignment software version 7: improvements in performance and usability. Mol Biol Evol. 2013:30:772-80.

19. Tanabe AS. Kakusan4 and Aminosan: two programs for comparing nonpartitioned, proportional and separate models for combined molecular phylogenetic analyses of multilocus sequence data. Mol Ecol Resour. 2011;11:914-21.

20. Tamura K, Peterson D, Peterson N, Stecher G, Nei M, Kumar S. MEGA5: molecular evolutionary genetics analysis using maximum likelihood, evolutionary distance, and maximum parsimony methods. Mol Biol Evol. 2011;28:2731-9.

21. Rozanov M, Plikat U, Chappey C, Kochergin A, Tatusova T. A web-based genotyping resource for viral sequences. Nucleic Acids Res. 2004:32:W654-659.

22. Tanaka Y, Sato Y, Osawa S, Inoue M, Tanaka S, Sasaki T. Suppression of feline coronavirus replication in vitro by cyclosporin A. Vet Res. 2012;43:41.

23. Simpson EH. Measurement of diversity. Nature. 1949;163:688.

24. Pielou EC. The measurement of diversity in different types of biological collections. J Theor Biol. 1966;11:225-8.

25. Herrewegh AA, Smeenk I, Horzinek MC, Rottier PJ, de Groot RJ. Feline coronavirus type II strains 79-1683 and 79-1146 originate from a double recombination between feline coronavirus type I and canine coronavirus. J Virol. 1998:72:4508-14.

26. Woo PC, Huang Y, Lau SK, Yuen KY. Coronavirus genomics and bioinformatics analysis. Viruses. 2010;2:1804-20

27. Tekes G, Hofmann-Lehmann R, Stallkamp I, Thiel V, Thiel HJ. Genome organization and reverse genetic analysis of a type I feline coronavirus. J Virol. 2008:82:1851-9.

28. Zhang X, Hasoksuz M, Spiro D, Halpin R, Wang S, Stollar S, et al. Complete genomic sequences, a key residue in the spike protein and deletions in nonstructural protein $3 \mathrm{~b}$ of US strains of the virulent and attenuated coronaviruses, transmissible gastroenteritis virus and porcine respiratory coronavirus. Virology. 2007:358:424-35.

29. Vlasova AN, Halpin R, Wang S, Ghedin E, Spiro DJ, Saif LJ. Molecular characterization of a new species in the genus Alphacoronavirus associated with mink epizootic catarrhal gastroenteritis. J Gen Virol. 2011;92:1369-79. 
30. An DJ, Jeoung HY, Jeong W, Park JY, Lee MH, Park BK. Prevalence of Korean cats with natural feline coronavirus infections. Virol J. 2011;8:455.

31. Benetka V, Kolodziejek J, Walk K, Rennhofer M, Mostl K. M gene analysis of atypical strains of feline and canine coronavirus circulating in an Austrian animal shelter. Vet Rec. 2006;159:170-4.

32. Duarte A, Fernandes M, Santos N, Tavares L. Virological Survey in free-ranging wildcats (Felis silvestris) and feral domestic cats in Portugal. Vet Microbiol. 2012;158:400-4.

33. Duarte A, Veiga I, Tavares L. Genetic diversity and phylogenetic analysis of Feline Coronavirus sequences from Portugal. Vet Microbiol. 2009;138:163-8.

34. Lin CN, Su BL, Wang CH, Hsieh MW, Chueh TJ, Chueh LL. Genetic diversity and correlation with feline infectious peritonitis of feline coronavirus type and II: a 5-year study in Taiwan. Vet Microbiol. 2009:136:233-9.

35. Ntafis V, Xylouri E, Mari V, Papanastassopoulou M, Papaioannou N, Thomas A, et al. Molecular characterization of a canine coronavirus NA/09 strain detected in a dog's organs. Arch Virol. 2012;157:171-5.

36. Soma T, Wada M, Taharaguchi S, Tajima T. Detection of ascitic feline coronavirus RNA from cats with clinically suspected feline infectious peritonitis. J Vet Med Sci. 2013;75:1389-92.

37. Battilani M, Coradin T, Scagliarini A, Ciulli S, Ostanello F, Prosperi S, et al. Quasispecies composition and phylogenetic analysis of feline coronaviruses (FCoVs) in naturally infected cats. FEMS Immunol Med Microbiol. 2003;39:141-7.

38. Gunn-Moore DA, Gunn-Moore FJ, Gruffydd-Jones TJ, Harbour DA. Detection of FcoV quasispecies using denaturing gradient gel electrophoresis. Vet Microbiol. 1999:69:127-30.

39. Herrewegh AA, Mahler M, Hedrich HJ, Haagmans BL, Egberink HF, Horzinek $M C$, et al. Persistence and evolution of feline coronavirus in a closed cat-breeding colony. Virology. 1997;234:349-63.

40. Kennedy MA, Moore E, Wilkes RP, Citino SB, Kania SA. Analysis of genetic mutations in the $7 \mathrm{a} 7 \mathrm{~b}$ open reading frame of coronavirus of cheetahs (Acinonyx jubatus). Am J Vet Res. 2006;67:627-32.

41. Kiss I, Kecskemeti S, Tanyi J, Klingeborn B, Belak S. Preliminary studies on feline coronavirus distribution in naturally and experimentally infected cats. Res Vet Sci. 2000;68:237-42.

42. Barker EN, Tasker S, Gruffydd-Jones TJ, Tuplin CK, Burton K, Porter E, et al. Phylogenetic analysis of feline coronavirus strains in an epizootic outbreak of feline infectious peritonitis. J Vet Intern Med. 2013;27:445-50.

43. Chang HW, de Groot RJ, Egberink HF, Rottier PJ. Feline infectious peritonitis: insights into feline coronavirus pathobiogenesis and epidemiology based on genetic analysis of the viral 3c gene. J Gen Virol. 2010;91:415-20.

44. Chang HW, Egberink HF, Rottier PJ. Sequence analysis of feline coronaviruses and the circulating virulent/avirulent theory. Emerg Infect Dis. 2011;17:744-6.

45. de Groot RJ, Baker SC, Baric RS, Brown CS, Drosten C, Enjuanes L, et al. Middle East respiratory syndrome coronavirus (MERS-CoV): announcement of the Coronavirus Study Group. J Virol. 2013;87:7790-2.

46. Lee $N$, Hui D, Wu A, Chan P, Cameron P, Joynt GM, et al. A major outbreak of severe acute respiratory syndrome in Hong Kong. $N$ Engl J Med. 2003:348:1986-94.

47. East ML, Moestl K, Benetka V, Pitra C, Honer OP, Wachter B, et al. Coronavirus infection of spotted hyenas in the Serengeti ecosystem. Vet Microbiol. 2004:102:1-9.

48. Kennedy M, Citino S, Dolorico T, McNabb AH, Moffat AS, Kania S. Detection of feline coronavirus infection in captive cheetahs (Acinonyx jubatus) by polymerase chain reaction. J Zoo Wildl Med. 2001;32:25-30.

49. Vijaykrishna D, Smith GJ, Zhang JX, Peiris JS, Chen H, Guan Y. Evolutionary insights into the ecology of coronaviruses. J Virol. 2007;81:4012-20.

\section{Submit your next manuscript to BioMed Central and take full advantage of:}

- Convenient online submission

- Thorough peer review

- No space constraints or color figure charges

- Immediate publication on acceptance

- Inclusion in PubMed, CAS, Scopus and Google Scholar

- Research which is freely available for redistribution

Submit your manuscript at www.biomedcentral.com/submit 